\title{
THE 2-DIMENSIONAL RIGIDITY OF CERTAIN FAMILIES OF GRAPHS
}

\author{
BILL JACKSON, BRIGITTE SERVATIUS, AND HERMAN SERVATIUS
}

\begin{abstract}
Laman's characterization of minimally rigid 2-dimensional generic frameworks gives a matroid structure on the edge set of the underlying graph, as was first pointed out and exploited by L. Lovász and Y. Yemini. Global rigidity has only recently been characterized by a combination of two results due to T. Jordán and the first named author, and R. Connelly, respectively. We use these characterizations to investigate how graph theoretic properties such as transitivity, connectivity and regularity influence (2-dimensional generic) rigidity and global rigidity and apply some of these results to reveal rigidity properties of random graphs. In particular, we characterize the globally rigid vertex transitive graphs, and show that a random $d$-regular graph is asymptotically almost surely globally rigid for all $d \geq 4$.
\end{abstract}

\section{INTRODUCTION}

We will use the term graph to denote a finite, undirected graph without loops or multiple edges. We use the term multigraph if loops and multiple edges are allowed. A (2-dimensional) framework $(G, p)$ is a pair $(G, p)$, where $G=(V, E)$ is a graph and $p$ is a map from $V$ to $\mathbb{R}^{2}$. We consider the framework to be a straight line embedding of the graph $G$ in $\mathbb{R}^{2}$. A framework $(G, p)$ is generic if the coordinates of all the points $p(v), v \in V$, are algebraically independent. Two frameworks $(G, p)$ and $(G, q)$ are equivalent if $\|p(u)-p(v)\|=\|q(u)-q(v)\|$ for all $u v \in E$. They are congruent if $\|p(u)-p(v)\|=\|q(u)-q(v)\|$ for all $u, v \in V$. The graph $G$ is globally rigid if every framework $(G, q)$ which is equivalent to a generic framework $(G, p)$, is in fact congruent to $(G, p)$. The graph $G$ is rigid if there exists an $\epsilon>0$ such that every framework $(G, q)$ which is equivalent to a generic framework $(G, p)$ and satisfies $\|p(u)-q(u)\|<\epsilon$ for all $u \in V$, is congruent to $(G, p)$. We say that $G$ is minimally rigid if $G$ is rigid, and $G-e$ is not rigid for all $e \in E$. Minimally rigid graphs were characterized by G. Laman in 1970.

Theorem 1.1. 10] A graph $G=(V, E)$ is minimally rigid if and only if (a) $|E|=2|V|-3$;

(b) $|F| \leq 2|V(F)|-3$ for all non-empty subsets $F$ of $E$ (where $V(F)$ denotes the support of $F$, i.e. the collection of all end points of the edges in $F$ ).

One may interpret condition (a) as requiring that $E$ contain enough edges to be rigid and condition (b) as requiring that none of these edges be "wasted" by packing too many between the vertices of any subset of $V$.

It can be seen that the edge sets satisfying conditions (a) and (b) satisfy the base axioms for a matroid on the edge set of the complete graph on the vertex set $V$, whose independent sets are the edge sets satisfying condition (b), see [6, 18. The rigidity matroid of a graph $G$ is the restriction of this matroid to the edge set 
of $G$. We will denote the rigidity matroid of $G$ by $\mathcal{M}(G)$ and its rank by $r(G)$. Thus $G=(V, E)$ is rigid if and only if $r(G)=2|V|-3$. We call the maximal rigid subgraphs of $G$ the rigid components of $G$. It is known that the edge sets of the rigid components of $G$ partition $E$ and that $\mathcal{M}(G)$ is the direct sum over its restrictions on the rigid components of $G$.

Theorem 1.1 was used by L. Lovasz and Y. Yemini [12] in 1982 to determine the rank function of the rigidity martoid of a graph. In particular they obtain the following characterisation of when a graph is rigid.

Theorem 1.2. 12 Let $G=(V, E)$ be a graph. Then $G$ is rigid if and only if for all families of induced subgraphs $\left\{G_{i}=\left(V_{i}, E_{i}\right)\right\}_{i=1}^{m}$ such that $E=\cup_{i=1}^{m} E_{i}$ we have $\sum_{i=1}^{m}\left(2\left|V_{i}\right|-3\right) \geq 2|V|-3$.

Let $k$ be a positive integer. A graph $G=(V, E)$ is $k$-connected if for all pairs of subgraphs $G_{1}, G_{2}$ of $G$ such that $G=G_{1} \cup G_{2},\left|V\left(G_{1}\right)-V\left(G_{2}\right)\right| \geq 1$ and $\left|V\left(G_{2}\right)-V\left(G_{1}\right)\right| \geq 1$, we have $\left|V\left(G_{1}\right) \cap V\left(G_{2}\right)\right| \geq k$.

A graph $G=(V, E)$ is redundantly rigid if $G-e$ is rigid for all $e \in E$, i.e. the removal of a single edge $e$ from the rigid graph $G$ does not destroy rigidity. Redundant rigidity also, just like rigidity, induces an equivalence relation on the edge set, whose equivalence classes are either maximal redundantly rigid subgraphs, or are singleton edges, called the redundantly rigid components. Again $\mathcal{M}(G)$ is the direct sum over its restrictions on the redundantly rigid components of $G$. Hendrickson [7] showed that redundant rigidity and 3-connectivity are necessary conditions for a graph to be globally rigid and conjectured that they are also sufficient. This conjecture was recently solved by R. Connelly, 3, T. Jordán and the first named author, 8 .

Theorem 1.3. [8] A graph $G$ is globally rigid if and only if $G$ is a complete graph on at most three vertices, or $G$ is both 3-connected and redundantly rigid.
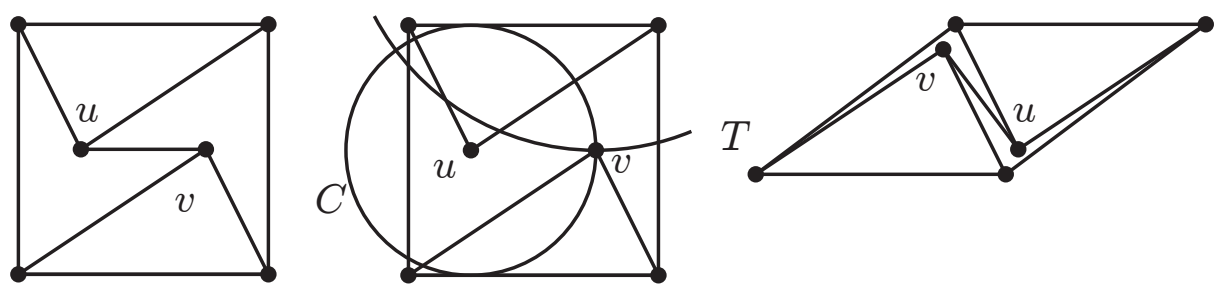

Figure 1. Finding an equivalent framework.

Figure 1 indicates the major difficulty with rigid but not globally rigid graphs: The edge lengths of such a framework do not uniquely determine the framework up to congruence, since the removal of a non-redundant edge $u v$ yields a mechanism, that is, a non-rigid framework with one degree of freedom. If we hold a rigid subgraph containing $u$ fixed (to mod out the isometries of the plane), the other endpoint $v$ of the removed edge can move on a trajectory $T$ which intersects a circle $C$ centered at $u$ whose radius is the length of $u v$ at least twice. Such situations pose computational difficulties in applied problems such as CAD or robotics. Global rigidity is the highly preferred property for these geometric applications and we will 
show that the troublesome rigid but not globally rigid graphs are rare exceptions in the family of vertex transitive graphs.

Lovász and Yemini [12] used Theorem 1.2 to show that every 6-connected graph $G$ is rigid, and note that their proof technique will show that $G-\left\{e_{1}, e_{2}, e_{3}\right\}$ is rigid for all $e_{1}, e_{2}, e_{3} \in E$, and hence that $G$ is redundantly rigid. This result was combined with Thoerem 1.3 in 8 to deduce

Theorem 1.4. 8 Every 6-connected graph is globally rigid.

We use Theorems 1.2 and 1.3 to investigate how other graph theoretic properties such as transitivity, cyclic connectivity and regularity influence rigidity and global rigidity of graphs. We apply some of these results to reveal rigidity properties of random graphs. In particular, we characterize the globally rigid vertex transitive graphs, and show that a random $d$-regular graph is asymptotically almost surely globally rigid for all $d \geq 4$.

We close this section with a rather technical lemma which we will use in applications of Theorem 1.2 .

Lemma 1.5. Let $\left(a_{1}, a_{2}, \ldots, a_{t}\right)$ be a non-increasing sequence of integers such that $t \geq 2$, and $a_{t} \geq 2$. Let $d=\sum_{i=1}^{t}\left(a_{i}-1\right)$ and

$$
g\left(a_{1}, a_{2}, \ldots, a_{t}\right):=\sum_{i=1}^{t}\left(2-\frac{3}{a_{i}}\right) .
$$

Then $g$ is an increasing function of $a_{1}$. Furthermore:

(a) $g\left(a_{1}, a_{2}, a_{3}, \ldots, a_{t-1}, a_{t}\right) \geq g\left(a_{1}+1, a_{2}, a_{3}, \ldots, a_{t-1}, a_{t}-1\right)$,

(b) $g\left(a_{1}, a_{2}, a_{3}, \ldots, a_{t-1}, 2\right) \geq g\left(a_{1}+1, a_{2}, a_{3}, \ldots, a_{t-1}\right)$,

(c) if $d \geq 4$ and $\left(a_{1}, a_{2}, \ldots, a_{t}\right) \notin\{(5,2),(4,2)\}$ then $g\left(a_{1}, a_{2}, \ldots, a_{t}\right) \geq 2$.

Proof. The proofs of (a) and (b) are straightforward. Using (a) and (b) we have $g\left(a_{1}, a_{2}, \ldots, a_{t}\right) \geq g(d, 2)=2-\frac{3}{d}+\frac{1}{2}$. Thus $g\left(a_{1}, a_{2}, \ldots, a_{t}\right) \geq 2$ when $d \geq 6$. If $d=$ 5 and $\left(a_{1}, a_{2}, \ldots, a_{t}\right) \neq(5,2)$ then $g\left(a_{1}, a_{2}, \ldots, a_{t}\right) \geq \min \{g(4,3), g(4,2,2)\}=\frac{9}{4}$. If $d=4$ and $\left(a_{1}, a_{2}, \ldots, a_{t}\right) \neq(4,2)$ then $g\left(a_{1}, a_{2}, \ldots, a_{t}\right) \geq \min \{g(3,3), g(3,2,2)\}=$ 2 .

\section{VERTEX TRANSITIVE GRAPHS}

We will characterize when vertex transitive graphs are rigid and globally rigid. Our characterization uses the following concept. A $k$-factor in a graph $G$ is a spanning $k$-regular subgraph. It also uses the following result which was obtained independently by W. Mader and M.E. Watkins.

Theorem 2.1. 14, 17 Let $G$ be a connected $k$-regular vertex transitive graph. Then the connectivity of $G$ is at least $\left\lceil\frac{2 k}{3}\right\rceil$.

Theorem 2.2. Let $G=(V, E)$ be a connected $k$-regular vertex transitive graph on $n$ vertices. Then $G$ is not globally rigid if and only if:

(a) $k=2$ and $n \geq 4$.

(b) $k=3$ and $n \geq 6$.

(c) $k=4$ and $G$ has a 3 -factor $F$ consisting of s disjoint copies of $K_{4}$ where $s \geq 3$.

(d) $k=5$ and $G$ has a 4-factor $F$ consisting of $s$ disjoint copies of $K_{5}$ where $s \geq 6$. 
Proof. We first suppose $G$ satisfies (a), (b), (c) or (d). Choose an edge $e \in E$ such that $e \notin E(F)$ in cases (c) and (d). Then we may use Theorem 1.2 to deduce that $G-e$ is not rigid. (In cases (a) and (b) we take the partition of $E-e$ consisting of copies of $K_{2}$, in case (c) we take the partition of $G-e$ consisting of the copies of $K_{4}$ in $F$ and the remaining copies of $K_{2}$, in case (d) we take the partition of $G-e$ consisting of the copies of $K_{5}$ in $F$ and the remaining copies of $K_{2}$.) Thus $G$ is not redundantly rigid and hence not globally rigid by Theorem 1.3 .

Suppose $G$ is not globally rigid. Then $G$ is not complete and hence if $k \leq 3$ we have (a) or (b). Thus we may assume $k \geq 4$. Using Theorem 2.1, we may deduce that $G$ is 4 -connected. Since $G$ is not globally rigid, it follows from Theorem 1.3 that $G$ is not redundantly rigid. Let $G_{i}=\left(V_{i}, E_{i}\right), 1 \leq i \leq m$ be the redundantly rigid components of $G$.

\section{Claim 2.3.}

$$
\sum_{v \in V} \sum_{V_{i} \ni v}\left(2-\frac{3}{\left|V_{i}\right|}\right) \leq 2 n-3 .
$$

Proof Since each $G_{i}$ is rigid we have $r\left(G_{i}\right)=2\left|V_{i}\right|-3$. Since $\mathcal{M}(G)=\oplus_{i=1}^{m} \mathcal{M}\left(G_{i}\right)$, we have

$$
\begin{aligned}
\sum_{v \in V} \sum_{V_{i} \ni v}\left(2-\frac{3}{\left|V_{i}\right|}\right)=\sum_{i=1}^{m} \sum_{v \in V_{i}}\left(2-\frac{3}{\left|V_{i}\right|}\right) & =\sum_{i=1}^{m}\left|V_{i}\right|\left(2-\frac{3}{\left|V_{i}\right|}\right) \\
& =\sum_{i=1}^{m}\left(2\left|V_{i}\right|-3\right)=r(G) \leq 2 n-3 .
\end{aligned}
$$

For $v \in V$, let the type of $v$ be the non-increasing sequence of integers given by the sizes of the redundantly rigid components which contain $v$. Since $G$ is vertex transitive each vertex of $G$ has the same type, say $\left(a_{1}, a_{2}, \ldots, a_{t}\right)$. We shall refer to this sequence as the type of $G$. Since $G$ is connected and has at least two redundantly rigid components, some vertex belongs to at least two redundantly rigid components so $t \geq 2$.

Claim 2.4. $G$ has type $(4,2)$ or $(5,2)$.

Proof: Suppose $G$ has type $\left(a_{1}, a_{2}, \ldots, a_{t}\right) \notin\{(4,2),(5,2)\}$. We have $a_{i} \geq 2$ and $4 \leq k \leq d=\sum_{i=1}^{t}\left(a_{i}-1\right)$. Lemma 1.5 now implies that $\sum_{V_{i} \ni v}\left(2-\frac{3}{\left|V_{i}\right|}\right) \geq 2$. Thus $\sum_{v \in V} \sum_{V_{i} \ni v}\left(2-\frac{3}{\left|V_{i}\right|}\right) \geq 2 n$. This contradicts Claim 2.3 .

We first suppose $G$ has type $(4,2)$. Then each vertex of $G$ belongs to exactly two redundantly rigid components with four and two vertices, respectively. Since $k \geq 4$, these components must be isomorphic to $K_{4}$ and $K_{2}$, respectively. Thus $k=4$ and $G$ has a factor $F$ consisting of $s$ disjoint copies of $K_{4}$. Furthermore, $G$ has $s$ redundantly rigid components isomorphic to $K_{4}$ and $2 s$ redundantly rigid components isomorphic to $K_{2}$. Since $n=4 s$, we can now use the inequality $2 n-3 \geq$ $r(G)=\sum_{i=1}^{m} r\left(G_{i}\right)$ to deduce that $s \geq 3$. Thus (c) holds for $G$.

Finally we consider the case when $G$ has type $(5,2)$. Then each vertex of $G$ belongs to exactly two redundantly rigid components with five and two vertices, respectively. Let $H$ be a rigid component of $G$ with five vertices. Since $G$ is $k$ regular, $H$ is $(k-1)$-regular. The fact that $k \geq 4$ and $|V(F)|=5$ now implies that $k=5$ and $H$ is isomorphic to $K_{5}$. Thus $k=5$ and $G$ has a factor $F$ consisting of $s$ disjoint copies of $K_{5}$. We can now deduce that $s \geq 6$ by again using the inequality $2 n-3 \geq r(G)=\sum_{i=1}^{m} r\left(G_{i}\right)$. Thus (d) holds for $G$. 
Corollary 2.5. Let $G=(V, E)$ be a connected $k$-regular vertex transitive graph on $n$ vertices. Then $G$ is not rigid if and only if:

(a) $k=2$ and $n \geq 4$.

(b) $k=3$ and $n \geq 8$.

(c) $k=4$ and $G$ has a factor $F$ consisting of $s$ disjoint copies of $K_{4}$ where $s \geq 4$.

(d) $k=5$ and $G$ has a factor $F$ consisting of $s$ disjoint copies of $K_{5}$ where $s \geq 8$.

Proof. If $G$ belongs to the families described in (a), (b), (c) and (d), then we can use use Theorem 1.2 to show that $G$ is not rigid.

To prove the reverse implication we suppose that $G$ is not rigid. Then $G$ is not complete so $n \geq 4$ if $k=2$ and $n \geq 6$ if $k=3$. Furthermore, there are exactly two vertex transitive 3-regular graphs on six vertices, $K_{3,3}$ and the triangular prism, and both are rigid. Thus $n \geq 8$ when $k=3$ and we may assume that $k \geq 4$. Since $G$ is not rigid, it is not globally rigid. Thus $G$ has type $(4,2)$ or $(5,2)$ and belongs to one of the families listed in Theorem 2.2 (c),(d). We can now use the analysis in the final part of the proof of Theorem 2.2 and the fact that $r(G) \leq 2|V|-4$ to deduce that $s \geq 4$ when $k=4$ and $s \geq 8$ when $k=5$.

Mader [15] has shown that if $G$ is a connected $k$-regular vertex transitive graph then $G$ is $k$-edge-connected. Tindell [16] extended this by showing that if $G$ has a non-trivial $k$-edge-cut, then $G$ has a (k-1)-factor consisting of disjoint copies of $K_{k}$ and the graph obtained from $G$ by contracting each of these $K_{k}$ 's to single vertices is a $k$-regular 1-arc-transitive multigraph. It follows that the graphs described in families (c) and (d) of Theorem 2.2 and Corollary 2.5 have this 'product' structure. Since the graph $C_{3}^{2}$ obtained by replacing each edge of a 3-cycle by two parallel edges is the only 4-regular 1-arc-transitive multigraph on three vertices, and $K_{6}$ is the only 5 -regular 1-arc-transitive multigraph on six vertices, we may deduce:

Corollary 2.6. There are exactly four vertex transitive graphs which are rigid but not globally rigid. These are $K_{3,3}$, the triangular prism, the graph, $G_{1}$, obtained from $C_{3}^{2}$ by replacing each vertex by a copy of $K_{4}$ and the graph, $G_{2}$, obtained from $K_{6}$ by replacing each vertex by a copy of $K_{5}$.
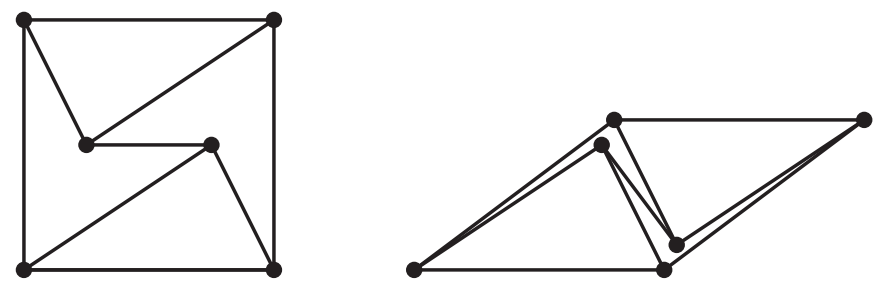

Figure 2. Two equivalent but non-congruent frameworks on the triangular prism.

\section{Highly CONNECTED GRAPHS}

We adapt the proof technique of Lovasz and Yemini in 12 to show that a slightly weaker connectivity hypothesis than that given in Theorem 1.4 is sufficient to imply global rigidity. We say $G$ is essentially 6 -connected if:

(a) $G$ is 4-connected, 

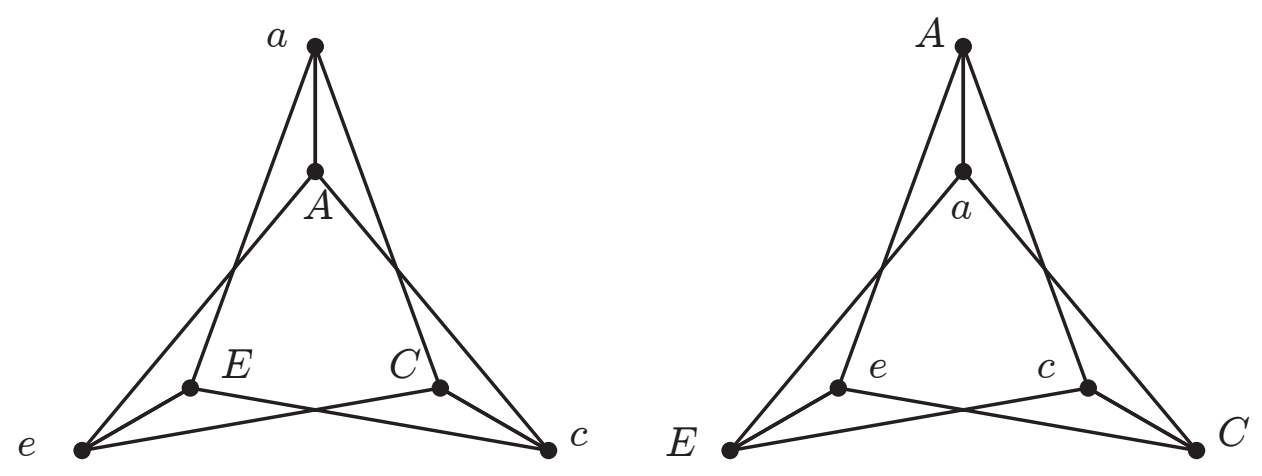

Figure 3. Two equivalent frameworks on $K_{3,3}$ for which there is no label preserving congruence.
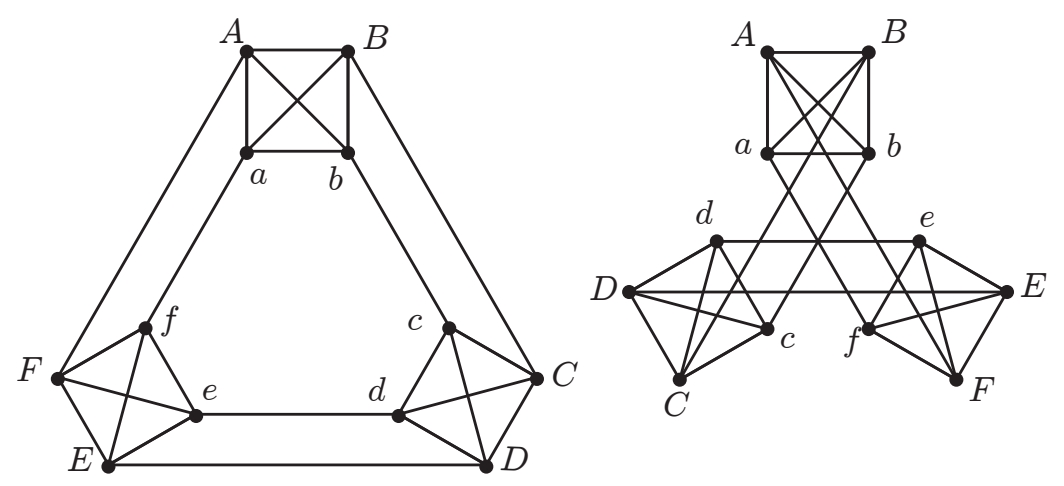

Figure 4. Two equivalent but non-congruent frameworks on $G_{1}$.
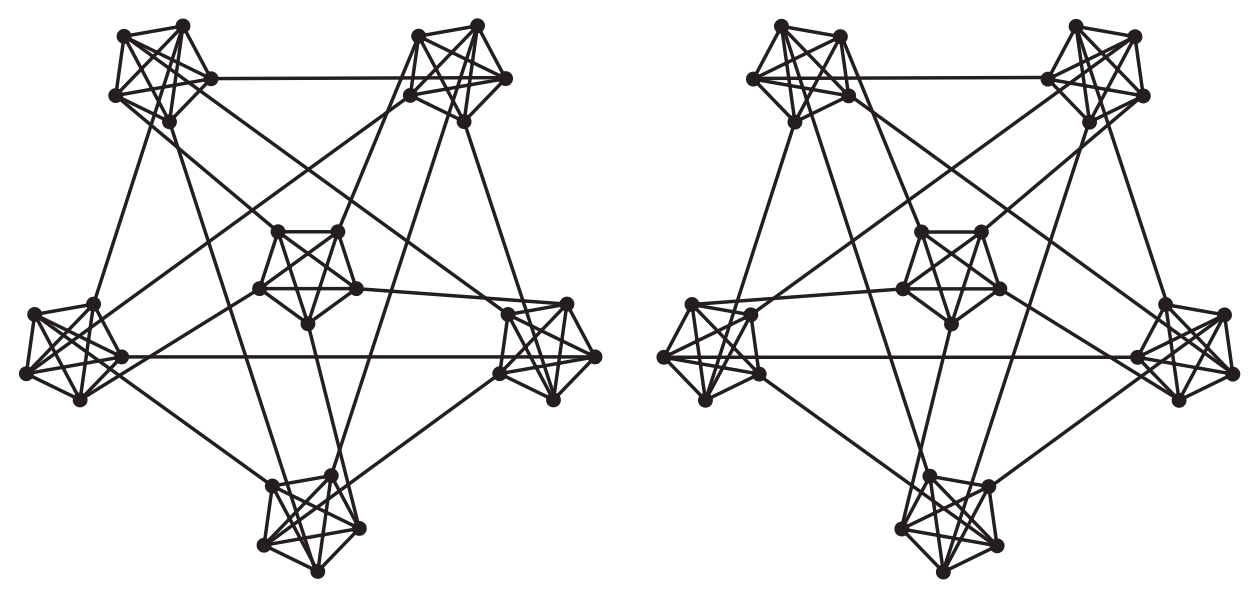

Figure 5. Two equivalent but non-congruent frameworks on $G_{2}$.

(b) for all pairs of subgraphs $G_{1}, G_{2}$ of $G$ such that $G=G_{1} \cup G_{2}, \mid V\left(G_{1}\right)$ $V\left(G_{2}\right) \mid \geq 3$ and $\left|V\left(G_{2}\right)-V\left(G_{1}\right)\right| \geq 3$, we have $\left|V\left(G_{1}\right) \cap V\left(G_{2}\right)\right| \geq 5$, and 
(c) for all pairs of subgraphs $G_{1}, G_{2}$ of $G$ such that $G=G_{1} \cup G_{2}, \mid V\left(G_{1}\right)$ $V\left(G_{2}\right) \mid \geq 4$ and $\left|V\left(G_{2}\right)-V\left(G_{1}\right)\right| \geq 4$, we have $\left|V\left(G_{1}\right) \cap V\left(G_{2}\right)\right| \geq 6$.

We shall first show that every essentially 6 -connected graph is redundantly rigid.

Theorem 3.1. Every essentially 6 -connected graph is redundantly rigid.

Proof. We proceed by contradiction. Suppose the theorem is false and let $G=$ $(V, E)$ be a counterexample chosen such that $|V|$ is as small as possible and, subject to this condition, $|E|$ is as large as possible. Choose an edge $e=x y \in E$ such that $G-e$ is not rigid. By Theorem 1.2 there exists a family of induced subgraphs $\left\{G_{i}=\left(V_{i}, E_{i}\right)\right\}_{i=1}^{m}$ such that $E-e=\cup_{i=1}^{m} E_{i}$ and $\sum_{i=1}^{m}\left(2\left|V_{i}\right|-3\right) \leq 2|V|-4$. Let $G_{0}$ be the subgraph of $G$ induced by $e$. Then $E=\cup_{i=0}^{m} E_{i}$ and

$$
\sum_{i=1}^{m}\left(2\left|V_{i}\right|-3\right) \leq 2|V|-3
$$

The maximality of $|E|$ implies that each subgraph $G_{i}, 0 \leq i \leq m$, is complete. For each $v \in V$ let $c(v)$ be the number of subgraphs $G_{i}$ containing $v$.

Claim 3.2. $c(v) \geq 2$ for all $v \in V$.

Proof: Suppose $c(v)=1$ for some $v \in V$. Clearly $v \notin V_{0}$. Without loss of generality, $v \in V_{m}$. Let $G^{\prime}=G-v, G_{i}^{\prime}=G_{i}$ for $1 \leq i \leq m-1$ and $G_{m}^{\prime}=G_{m}-v$. Then $E^{\prime}-e=\cup_{i=1}^{m} E_{i}^{\prime}$ and

$$
\sum_{i=1}^{m}\left(2\left|V_{i}^{\prime}\right|-3\right)=\sum_{i=1}^{m}\left(2\left|V_{i}\right|-3\right)-2 \leq 2|V|-4=2\left|V^{\prime}\right|-4 .
$$

Thus $G^{\prime}-e$ is not rigid by Theorem 1.2 The minimality of $|V|$ implies that $G^{\prime}$ is not essentially 6-connected. Suppose $H_{1}^{\prime}, H_{2}^{\prime}$ are subgraphs of $G^{\prime}$ such that $G^{\prime}=H_{1}^{\prime} \cup H_{2}^{\prime}$. Then the fact that $N_{G}(v)$ induces a complete graph in $G^{\prime}$ implies that $N_{G}(v) \subseteq V\left(H_{1}^{\prime}\right)$ or $N_{G}(v) \subseteq V\left(H_{2}^{\prime}\right)$. By symmetry $N_{G}(v) \subseteq V\left(H_{1}^{\prime}\right)$. Let $H_{1}$ be the subgraph of $G$ induced by $V\left(H_{1}^{\prime}\right) \cup\{v\}$ and $H_{2}=H_{2}^{\prime}$. Hence, if we choose $H_{1}^{\prime}, H_{2}^{\prime}$ to certify the fact that $G^{\prime}$ is not essentially 6 -connected, then $H_{1}, H_{2}$ will contradict the fact that $G$ is essentially 6-connected.

Claim 3.3.

$$
\sum_{v \in V} \sum_{V_{i} \ni v}\left(2-\frac{3}{\left|V_{i}\right|}\right) \leq 2|V|-3
$$

Proof: We have

$$
\sum_{v \in V} \sum_{V_{i} \ni v}\left(2-\frac{3}{\left|V_{i}\right|}\right)=\sum_{i=0}^{m} \sum_{v \in V_{i}}\left(2-\frac{3}{\left|V_{i}\right|}\right)=\sum_{i=0}^{m}\left|V_{i}\right|\left(2-\frac{3}{\left|V_{i}\right|}\right) \leq 2|V|-4
$$

by (1).

For $v \in V$ the type of $v$ is the non-increasing sequence of integers given by the sizes of the sets $V_{i}$ which contain $v$.

Claim 3.4. Suppose $v \in V$. Then $\sum_{V_{i} \ni v}\left(2-\frac{3}{\left|V_{i}\right|}\right) \geq 2$ unless $v$ has type $(4,2)$ or $(5,2)$.

Proof. Suppose $v$ has type $\left(a_{1}, a_{2}, \ldots, a_{t}\right)$. Since $G$ is 4-connected, $d=\sum_{i=1}^{t}\left(a_{i}-\right.$ $1) \geq 4$. The claim now follows from Lemma 1.5 
Claim 3.5. $|V| \geq 16$.

Proof. Using Claims 3.3, 3.4 and the facts that $g(4,2)=\frac{7}{4}$ and $g(5,2)=\frac{19}{10}$, we may deduce that

$$
\frac{7}{4}|V| \leq \sum_{v \in V} \sum_{V_{i} \ni v}\left(2-\frac{3}{\left|V_{i}\right|}\right) \leq 2|V|-4 .
$$

Thus $|V| \geq 16$.

We say that a vertex $v \in V$ is bad if it has type $(4,2)$ or $(5,2)$ and that a set $V_{i}$ is bad if it has size four or five and contains a bad vertex. Let $B$ be the family of all bad sets $V_{i}$. For each vertex $v \in V$ let $b(v)$ be the number of bad sets $X_{i} \in B$ which contain $v$. For $i=0,1$ let $U_{i}=\{v \in V: b(v)=i\}$ and put $U_{2}=V-\left(U_{0} \cup U_{1}\right)$. Using Claim 3.4 we may deduce

Claim 3.6. $\sum_{v \in U_{0}} \sum_{V_{i} \ni v}\left(2-\frac{3}{\left|V_{i}\right|}\right) \geq 2\left|U_{0}\right|$.

For $V_{j} \in B$ let $V_{j}^{\prime}=\left\{v \in V_{j}: b(v)=1\right\}$ and $V_{j}^{\prime \prime}=\left\{v \in V_{j}: b(v) \geq 2\right\}$.

Claim 3.7. For $V_{j} \in B$ we have

$$
\sum_{v \in V_{j}^{\prime}} \sum_{V_{i} \ni v}\left(2-\frac{3}{\left|V_{i}\right|}\right)+\sum_{v \in V_{j}^{\prime \prime}}\left(2-\frac{3}{\left|V_{j}\right|}\right) \geq 2\left|V_{j}^{\prime}\right|+\left|V_{j}^{\prime \prime}\right| .
$$

Proof: Since $V_{j} \in B$, we have $\left|V_{j}\right| \in\{4,5\}$. Let $A_{j}$ be the set of bad vertices in $V_{j}$. Then $A_{j} \subseteq V_{j}^{\prime}$ and $\left|A_{j}\right| \geq 1$ since $V_{j} \in B$. Consider the following two cases.

$\left|\boldsymbol{V}_{\boldsymbol{j}}\right|=4$. Condition (b) in the definition of essentially 6-connected and Claim 3.5 imply that $\left|A_{j}\right| \leq 2$. For $v \in A_{j}$ we have $\sum_{V_{i} \ni v}\left(2-\frac{3}{\left|V_{i}\right|}\right)=\frac{7}{4}$. For $v \in V_{j}^{\prime}-A_{j}$ Lemma 1.5 implies that $\sum_{V_{i} \ni v}\left(2-\frac{3}{\left|V_{i}\right|}\right) \geq \min \{g(4,3), g(4,2,2)\}=\frac{9}{4}$. These facts can be used to verify that the claim holds in each of the four subcases $\left|V_{j}^{\prime}\right| \in$ $\{1,2,3,4\}$.

$\left|\boldsymbol{V}_{\boldsymbol{j}}\right|=\mathbf{5}$. Condition (c) in the definition of essentially 6-connected and Claim 3.5 imply that $\left|A_{j}\right| \leq 3$. For $v \in A_{j}$ we have $\sum_{V_{i} \ni v}\left(2-\frac{3}{\left|V_{i}\right|}\right)=\frac{19}{10}$. For $v \in V_{j}^{\prime}-A_{j}$ use Lemma 1.5 implies that $\sum_{V_{i} \ni v}\left(2-\frac{3}{\left|V_{i}\right|}\right) \geq \min \{g(5,3), g(5,2,2)\}=\frac{12}{5}$. These facts can be used to verify that the claim holds in each of the five subcases $\left|V_{j}^{\prime}\right| \in$ $\{1,2,3,4,5\}$.

Claim 3.8. $\sum_{v \in U_{1} \cup U_{2}} \sum_{V_{i} \ni v}\left(2-\frac{3}{\left|V_{i}\right|}\right) \geq 2\left|U_{1}\right|+2\left|U_{2}\right|$.

Proof: We have

$$
\begin{aligned}
\sum_{v \in U_{1} \cup U_{2}} \sum_{V_{i} \ni v}\left(2-\frac{3}{\left|V_{i}\right|}\right) & =\sum_{v \in U_{1}} \sum_{V_{i} \ni v}\left(2-\frac{3}{\left|V_{i}\right|}\right)+\sum_{v \in U_{2}} \sum_{V_{i} \ni v}\left(2-\frac{3}{\left|V_{i}\right|}\right) \\
& \geq \sum_{V_{j} \in B} \sum_{v \in V_{j}^{\prime}} \sum_{V_{i} \ni v}\left(2-\frac{3}{\left|V_{i}\right|}\right)+\sum_{V_{j} \in B} \sum_{v \in V_{j}^{\prime \prime}}\left(2-\frac{3}{\left|V_{j}\right|}\right) \\
& \geq \sum_{V_{j} \in B}\left(2\left|V_{j}^{\prime}\right|+\left|V_{j}^{\prime \prime}\right|\right)
\end{aligned}
$$

by Claim 3.7. Since each vertex in $U_{1}$ belongs to exactly one set $V_{j}^{\prime}$ and each vertex in $U_{2}$ belongs to at least two distinct sets $V_{j}^{\prime \prime}$, we have $\sum_{V_{j} \in B}\left(2\left|V_{j}^{\prime}\right|+\left|V_{j}^{\prime \prime}\right|\right) \geq$ $2\left|U_{1}\right|+2\left|U_{2}\right|$. 
We can now complete the proof of the theorem. Claims 3.6 and 3.8 give

$$
\sum_{v \in V} \sum_{V_{i} \ni v}\left[2-\frac{3}{\left|V_{i}\right|}\right] \geq 2|V|
$$

This contradicts Claim 3.3

Combining Theorem 3.1 with Theorem 1.3 , we immediately deduce

Theorem 3.9. Every essentially 6-connected graph is globally rigid.

As an example we note that the complete bipartite graph $K_{4, m}, m \geq 4$, satisfies the hypotheses of Theorem 3.9, and hence is globally rigid, but does not satisfy the hypotheses of Theorem 1.4

Our final result of this section observes that an even weaker connectivity condition is sufficient to imply that 4-regular graphs are globally rigid. A graph $G=(V, E)$ is said to be cyclically $k$-edge-connected if for all $X \subseteq V$ such that $G[X]$ and $G[V-X]$ both contain cycles, we have at least $k$ edges from $X$ to $V-X$.

Theorem 3.10. Let $G=(V, E)$ be a cyclically 5-edge-connected 4-regular graph. Then $G$ is globally rigid.

Proof. Suppose that $G$ is not globally rigid. The facts that $G$ is cyclically 5 -edgeconnected and 4-regular imply that $G$ is 3 -connected. Hence, by [8], $G$ is not redundantly rigid. Let $G_{1}=\left(V_{1}, E_{1}\right)$ be a largest redundantly rigid subgraph of $G$. Since $|E|=2 n, G$ contains an $M$-circuit and hence $\left|V_{1}\right| \geq 4$ and $\left|E_{1}\right| \geq 2\left|V_{1}\right|-2$. Since $G$ is 4-regular this implies that $d_{G}\left(V_{1}\right) \leq 4$ and $\left|E\left(G-V_{1}\right)\right| \geq 2\left|V-V_{1}\right|-2$. Thus $G_{1}$ and $G-G_{1}$ both contain cycles. Now the fact that $d_{G}\left(V_{1}\right) \leq 4$ contradicts the hypothesis that $G$ is cyclically 5 -edge-connected.

Note that examples of non-rigid 4-regular 4-connected graphs and non-rigid 5regular 5-connected graphs are given in Theorem 2.5 (c), (d).

\section{RANDOM GRAPHS}

Let $G_{n, d}$ denote the probability space of all $d$-regular graphs on $n$ vertices with the uniform probability distribution. A sequence of graph properties $A_{n}$ holds asymptotically almost surely, or a.a.s. for short, in $G_{n, d}$ if $\lim _{n \rightarrow \infty} \operatorname{Pr}_{G_{n, d}}\left(A_{n}\right)=$ 1. Graphs in $G_{n, d}$ are known to be a.a.s. highly connected. It was shown by Bollobás [1] and Wormald [19] that if $G \in G_{n, d}$ for any fixed $d \geq 3$, then $G$ is a.a.s. $d$-connected. This result was extended to all $3 \leq d \leq n-4$ by Cooper et al. 4 and Krivelevich et al. 9]. Stronger results hold if we discount 'trivial' cutsets. In [20], Wormald shows that if if $G \in G_{n, d}$ for any fixed $d \geq 3$, then $G$ is a.a.s. cyclically $(3 d-6)$-edge-connected. Together with Theorem 3.10 this immediately gives:

Theorem 4.1. If $G \in G_{n, 4}$ then $G$ is a.a.s. globally rigid.

In fact this result holds for all $d \geq 4$.

Theorem 4.2. If $G \in G_{n, d}$ and $d \geq 4$ then $G$ is a.a.s. globally rigid.

Proof. If $d \geq 6$ then $G$ is a.a.s. 6-connected by [4, 9] and the result follows from Theorem 1.4. If $d=5$ then the result follows from Theorem 4.1 by contiguity, see [20]. 
Let $G(n, p)$ denote the probability space of all graphs on $n$ vertices in which each edge is chosen independently with probability $p$. In the following we will assume that all logarithms are natural. We will need the following results on $G(n, p)$.

Lemma 4.3. Let $G \in G(n, p)$, where $p=(\log n+k \log \log n+w(n)) / n, k \geq 2$ is an integer and $\lim _{n \rightarrow \infty} w(n)=\infty$. For each fixed integer $t$, let $S_{t}$ be the set of vertices of $G$ of degree at most $t$. Then, a.a.s.

(a) $S_{k-1}$ is empty,

(b) no two vertices of $S_{t}$ are joined by a path of length at most two in $G$,

(c) $G-S_{t-1}$ is non-empty and t-connected.

Proof. Facts (a) and (b) are well known, see for example [2]. Fact (c) follows from (a), (b) and [13, Theorem 4]

Theorem 4.4. Let $G \in G(n, p)$, where $p=(\log n+k \log \log n+w(n)) / n$, and $\lim _{n \rightarrow \infty} w(n)=\infty$.

(a) If $k=2$ then $G$ is a.a.s. rigid.

(b) If $k=3$ then $G$ is a.a.s. globally rigid.

Proof. (a) We adopt the notation of Lemma 4.3. It follows from Lemma 4.3 that a.a.s. $S_{1}=\emptyset$ and $G-S_{5}$ is a.a.s. 6-connected. Hence $G-S_{5}$ is a.a.s. (globally) rigid by Theorem 1.4. Since adding a new vertex joined by at least two new edges to a rigid graph preserves rigidity, it follows that $G$ is a.a.s. rigid.

(b) This follows in similar way to (a), using the facts that $S_{2}=\emptyset$ and that adding a new vertex joined by at least three new edges to a globally rigid graph preserves global rigidity.

The bounds on $p$ given in Theorem 4.4 are best possible since if $G \in G(n, p)$ and $p=(\log n+k \log \log n+c) / n$ for any constant $c$, then $G$ does not a.a.s. have minimum degree at least $k$, see [2].

Let $\operatorname{Geom}(n, r)$ denote the probability space of all graphs on $n$ vertices in which the vertices are distributed uniformly at random in the unit square and each pair of vertices of distance at most $r$ are joined by an edge. Suppose $G \in \operatorname{Geom}(n, r)$. $\mathrm{Li}$, Wan and Wang [11] have shown that if $n \pi r^{2}=\log n+(2 k-3) \log \log n+w(n)$ for $k \geq 2$ a fixed integer and $\lim _{n \rightarrow \infty} w(n)=\infty$, then $G$ is a.a.s. $k$-connected. As noted by Eren et al. [5], this result can be combined with Theorem 1.4 to deduce that if $n \pi r^{2}=\log n+9 \log \log n+w(n)$ then $G$ is a.a.s. globally rigid. On the other hand, it is also shown in [1] that if $n \pi r^{2}=\log n+(k-1) \log \log n+c$ for any constant $c$, then $G$ is not a.a.s. $k$-connected. It is still conceivable, however, that if $n \pi r^{2}=\log n+\log \log n+w(n)$ then $G$ is a.a.s. rigid, and that if $n \pi r^{2}=$ $\log n+2 \log \log n+w(n)$ then $G$ is a.a.s. globally rigid.

\section{REFERENCES}

[1] B. BollobÁs, Random graphs, in Surveys in Combinatorics 1999 (N.V. Temperley, ed.), LMS Lecture Note Series 52, Cambridge University Press, Cambridge, UK, 1981, 80-102.

[2] B. Bollobás, Random graphs Academic Press, New York, 1985.

[3] R. Connelly, Generic Global Rigidity, Discrete Comput. Geom., to appear. http://www.springerlink.com/link.asp?id=TE8FBTNH8FGP

[4] C. Cooper, A. Frieze And B. Reed, Random regular graphs of non-constant degree, SIAM J. Algebraic Discrete Methods 3 (1982), no. 1, 91-98. 
[5] T. Eren, D.K. Goldenberg, W. Whiteley and Y.R. Yang, A.S. Morse, B.D.O. AnderSON, AND P.N. Belhumeur, Rigidity, computation, and randomization in network localization, preprint. http://citeseer.ist.psu.edu/eren04rigidity.html

[6] J. Graver, B. Servatius and H. Servatius Combinatorial rigidity, American Mathematical Society (1993).

[7] B. Hendrickson, Conditions for unique graph realizations, SIAM J. Comput. 21 (1992), no. $1,65-84$

[8] B. JACKSON AND T. JORDÁN, Connected rigidity matroids and unique realizations of graphs, J. Comb. Th., Series B, to appear.

[9] M. Krivelevitch, B. Sudakov, V.H. Vu and N.C. Wormald, Random regular graphs of high degree, SIAM J. Algebraic Discrete Methods 3 (1982), no. 1, 91-98.

[10] G. Laman On Graphs and rigidity of plane skeletal structures, J. Engrg. Math. 4 (1970), $331-340$.

[11] X-Y. Li, P-J. WAN And Y. WAng, Fault tolerance deployment and topology control in wireless networks, in Proceedings of the ACM Symposium on Mobile Ad Hoc Networking and Computing (Mobihoc) (2003), Annapolis, MD, June 2003. http://www.sigmobile.org/mobihoc/2003/papers/p117-li.pdf

[12] L. Lovász And Y. Yemini, On generic rigidity in the plane, SIAM J. Algebraic Discrete Methods 3 (1982), no. 1, 91-98.

[13] T. LuCZAK, Size and connectivity of the $k$-core of a random graph, Discrete Math. 91 (1991), $61-68$.

[14] W. MADER, Über den Zusammenhang symmetrischer Graphen, Archiv. der Math. 21 (1970) $331-336$.

[15] W. Mader, Minimale n-fach kantenzusammenhängende Graphen, Math. Ann. 191 (1971) $21-28$.

[16] R. Tindell, Edge connectivity properties of symmetric graphs, Preprint, Stevens Institute of Technology, Hoboken, NJ (1982).

[17] M.E. Watkins, Connectivity of transitive graphs, J. Comb. Th. 8 (1970) 23-29.

[18] W. Whiteley, Some matroids from discrete applied geometry, Matroid theory (Seattle, WA, 1995), 171-311, Contemp. Math., 197, Amer. Math. Soc., Providence, RI, 1996.

[19] N.C. Wormald, The asymptotic connectivity of labelled regular graphs, J. Comb. Th., Series $B,(1981), 156-167$.

[20] N.C. Wormald, Models of random regular graphs, in Surveys in Combinatorics 1999 (J.D. Lamb and D.A. Preece, eds.), (1999), 239-298.

School of Mathematical Sciences, Queen Mary, University of London, Mile End RoAd, London E1 4NS, England

E-mail address: B.Jackson@qmul.ac.uk

Mathematical Sciences, Worcester Polytechnic Institute, Worcester MA 016092280.

E-mail address: bservat@wpi.edu

Dept. of Math and C.S, Clark University, Worcester, MA 01610-1477

E-mail address: hservatius@clarku.edu 